$86.7 \%$ of patients were satisfied with the process. The department is planning to increase the number of CAS clinics in future.

\section{P366 MANAGEMENT OF COMPLEX ENDOSCOPIC LESIONS THROUGH AN ADVANCED-ENDOSCOPY MULTIDISCIPLINARY TEAM-MEETING: EXPERIENCE FROM A TERTIARY CENTRE}

\begin{abstract}
${ }^{1}$ Alberto Murino, ${ }^{1}$ Nikolaos Lazaridis, ${ }^{1}$ Alexandros Skamnelos*, ${ }^{1}$ Nikolaos Koukias, ${ }^{1}$ Claudia Coppo, ${ }^{1}$ Deborah Costa, ${ }^{2}$ Massimo Varcada, ${ }^{3}$ Jennifer Watkins, ${ }^{4}$ Peter Wylie, ${ }^{3}$ TuVinh Luong, ${ }^{1}$ Regina Raymond, ${ }^{1}$ Alara Bailey, ${ }^{2}$ Colin Hart, ${ }^{4}$ Katie Planche, ${ }^{1}$ Edward J Despott. ${ }^{1}$ Royal Free Unit for Endoscopy, The Royal Free Hospital and University College London (UCL) Institute for Liver and Digestive Health, London, UK; ${ }^{2}$ Department of Colorectal Surgery, The Royal Free Hospital and University College London (UCL) Institute for Liver and Digestive Health, London, UK; ${ }^{3}$ Department of Cellular Pathology, The Royal Free Hospital and University College London (UCL) Institute for Liver and Digestive Health, London, UK; ${ }^{4}$ Department of Radiology, The Royal Free Hospital and University College London (UCL) Institute for Liver and Digestive Health, London, UK
\end{abstract}

\subsection{6/gutjnl-2020-bsgcampus.440}

Optimisation of patient care though multidisciplinary team (MDT) meetings is now established as the standard of care in cancer pathways. Cases presented at MDTs are discussed by a panel of experts in order to provide optimal management. An 'Advanced-endoscopy MDT' may help to achieve consensus on decision-making, clinical outcomes and patient satisfaction.

In our tertiary referral institution, we developed and adopted an 'Advanced-Endoscopy MDT' to evaluate complex cases. A bi-weekly meeting was established with the presence of expert interventional endoscopists, radiologists, histopathologists, dedicated gastrointestinal surgeons and nurse endoscopists. Depending on the cases discussed, additional experts were also in attendance. Endoscopic images, radiological scans and histopathology reports were assessed in order to decide treatment options and surveillance intervals. We retrospectively reviewed all patients that were discussed. Demographic, clinical, endoscopic findings and outcome data were analysed.

Over 20 months, 35 meetings were conducted; 463 cases were discussed for a total of 323 patients. The main reasons for MDT referral were for consideration of double-balloon enteroscopy (DBE) (107 patients) and for appropriate endoscopic or surgical management of mucosal/submucosal GI lesions. Through MDT discussion, 9 patients were referred for surgical management; 34 patients were treated with endoscopic submucosal dissection (ESD) and 72 patients with endoscopic mucosal resection (EMR). One ESD procedure was abandoned due to high suspicion of deep invasion and was referred for surgery. From the remaining 33 cases, R0 margins were reported for $32 / 33$ patients. Eight patients referred for DBE did not require endoscopic input and 29 cases were reviewed twice as additional radiological investigations or small bowel capsule endoscopy were deemed necessary. No major adverse event including perforation, severe delayed GI bleed or sepsis occurred after any endoscopic procedure discussed at the MDT.

Based on our preliminary experience of an 'AdvancedEndoscopy MDT' is a useful platform for the safe, effective and efficient management of complex advanced endoscopic procedures.

\section{P367 DEVELOPING THE GASTROENTEROLOGY/ENDOSCOPY WORKFORCE FOR 2025 AND BEYOND: IMPLICATIONS FOR ENDOSCOPY AND BOWEL CANCER SCREENING}

Vathsan Ravindran*, Ian Penman, Mark Coleman, Andy Veitch, Nicola Fearnhead, Robert PH Logan. King's College Hospital, London, London, UK

\subsection{6/gutjnl-2020-bsgcampus.441}

Introduction Delivering better outcomes for cancer is a major part of the Long Term Plan for the NHS. For colo-rectal cancer (CRC) better outcomes will mainly come from earlier diagnosis of CRC and polyps via the bowel cancer screening programme (BCSP). However more CRC screening has implications for the Bowel Cancer screening workforce. Currently there is no data to inform how much additional screening can be undertaken. The aim of this survey was to assess workforce career intentions with regards to screening and non-screening endoscopy.

Methods A questionnaire survey, developed by the core stakeholders, sent to all members of the BSG, ACPGB\&I and JAG between Feb-April 2020.

Results Responses were received from 352 endoscopists (age 25-74 yr old) most of whom worked in England. Respondents were non-screening colonoscopists (40\%), screening colonoscopists (24\%), nurse endoscopists (19\%) and trainees (17\%). Amongst current screening colonoscopists, most undertook 2 lists per week, one of which was for screening. However many were keen to do an additional screening list each week, if they were able to relinquish emergency care (71\%), ward cover $(45 \%)$ or an outpatient clinic (41\%). Importantly, $40 \%$ of the current screening workforce were planning to stop screening in the next 2-5 yrs due to retirement/pension issues. Of non-screening consultants 36\% were considering becoming JAG accredited if they could give up emergency care $(83 \%)$, ward cover $(67 \%)$ or an outpatient clinic (43\%). Interests outside of screening (42\%) was the most common reason for not wanting to become a screener. In addition, there was little enthusiasm for undertaking more endoscopy. However amongst trainees, $72 \%$ wanted to contribute to the BCSP, particularly if they did not participate in acute medicine or GIM ward cover. Their ideal job plan would have 1-2 BCSP lists per week, as did 48\% of the nurse endoscopists who wanted to become BSCP colonoscopists.

Conclusions Amongst the current consultant workforce there is some appetite to contribute to the BCSP, particularly if allowed to step away from emergency/acute care. However, the impact of early retirement and pensions needs to be considered within the plans to develop the screening workforce. Many trainees and nurse endoscopists would like to become BCSP colonoscopists.

\section{P368 SERVICE EVALUATION OF AN IBD HELP LINE}

Mohamed El-Naggar*, Sadek Malas, Anita D'Souza. Imperial College Healthcare NHS Trust, London, UK

\subsection{6/gutjnl-2020-bsgcampus.442}

Introduction Dedicated specialist advice lines for patients with IBD (Inflammatory bowel disease) are recommended by the BSG [Lamb CA, et al. Gut 2019;0:1-106] to help prioritise rapid access to advice and specialist clinical input. This project evaluated the efficacy of the service in a tertiary London 
hospital, particularly its role in managing patients concerns and avoiding hospital admission.

Methods A retrospective audit of 1000 IBD patient calls between August 2019 and October 2019 was conducted. The help line covered patients across all Imperial Trust sites - Charing Cross, St Mary's and Hammersmith Hospitals. The data collected included the following - Time of call, diagnosis, mode of contact, when the call was answered, number of attempts, advice given by whom, call reason and outcome.

Results The divide between UC and Crohn's was approximately equal $(46.3 \%$ vs $48.5 \%)$. Out of the 1000 the majority (809) were direct patient contact. Voicemail was the most common mode of contact (577) followed closely by email (435).

$84 \%$ of patients were answered on the same day, $12 \%$ the next day and $4 \%$ on another day. $84 \%$ of patients got through on the first attempt while $11 \%$ needed a second attempt and $5 \%$ required three attempts. Majority of the advice was given via telephone $(70 \%)$, the second most common was email (24\%).

Most calls (25\%) were regarding investigation/treatment, $21 \%$ regarding flares, $12 \%$ results, $11 \%$ admin, $11 \%$ advice, $7 \%$ homecare, $4 \%$ earlier appointment and 4\% side effects.

Regarding outcomes - $28 \%$ involved investigation/treatment, $13 \%$ repeat prescriptions, $13 \%$ results, $12 \%$ advice, $7 \%$ appointments, 5\% admin, 3\% dose escalation, 3\% home care, $3 \%$ contacted the consultant, $2 \%$ biologics switch, $2 \%$ A\&E/ urgent care referrals and $1 \%$ support.

Conclusions The service was highly efficient; $85 \%$ of calls were answered $<24$ hours while the clear majority could get through on the first attempt. A significant amount of patient contact was via email - allowing flexibility of contact between IBD Specialist nurses, patients and medical team.

The majority of calls and outcomes related to investigation, treatment and disease flare-up - demonstrating that the service is being used appropriately.

Only $2 \%$ of patients required A\&E/urgent care referrals, demonstrating that access to specialist advice can reduce or avoid costly hospital admissions. These data are in keeping with systematic reviews that have all shown advice lines to be safe and cost-effective. Medication advice and monitoring was a common use of the advice line - this remote service helps provide a robust platform for toxicity surveillance.

In order to maintain the high quality of the service, ongoing IBD Nurse education and prescribing, can help maintain high levels of efficiency, good patient care and a high level of patient satisfaction.

\section{P369 IMPLEMENTING A BIOLOGIC SWITCH PATHWAY TO FACILITATE TRANSITION TO BIOSIMILAR}

${ }^{1}$ Francesca Mastaglio*, ${ }^{2}$ Barbara Robertson, ${ }^{3}$ Diane Crake, ${ }^{4}$ Norma McConnell, ${ }^{5}$ Nicola Brownlee, ${ }^{1}$ Julie Fyall, ${ }^{1}$ Max Groome, ${ }^{1}$ John Todd, ${ }^{1}$ Jacqueline Paterson, ${ }^{1}$ Craig Mowat. 'Gastroenterology, NHS Tayside, Dundee, UK; ${ }^{2}$ Outpatents, NHS Tayside, Dundee, UK; ${ }^{3}$ Rheumatology, NHS Tayside, Dundee, UK; ${ }^{4}$ Administration and Clerical Service, NHS Tayside, Dundee, UK; ${ }^{5}$ Pharmacy, NHS Tayside, Dundee, UK

\subsection{6/gutjnl-2020-bsgcampus.443}

Introduction Biosimilar switches are likely to become a common scenario across specialties, can deliver significant savings, but can present a logistical dilemma. We implemented a Biologic Switch pathway focused on group Patient Education
Sessions (PES), devised by rheumatology colleagues, to facilitate a rapid patient-centred switch to biosimilar adalimumab in our IBD service.

Methods Adalimumab users were identified from IBD service excel spreadsheet of recorded users, Lloyds Pharmacy dispensing records and review of Clinic letters. Clerical support set up a 1 hour PES 'clinics' on the hospital patient management system (Trakcare); 10 patients per group session. Each patient was asked to record their preferences in an information letter explaining the switch and an optional invitation to attend the PES. A Registered General Nurse with no specialist knowledge of IBD was seconded for 10 weeks as a Biologics Switch nurse to deliver PES. On attendance, patients could ask the Biologic Switch Nurse questions about biosimilar, received pen device demonstration and tuition, a copy of manufacturer's booklet, Biologics Alert Card.

Results 138 patients were identified of which 132 were verified as receiving Humira and invited to attend a PES. 32 (24\%) patients were happy to switch without PES, 43 (33\%) patients accepted PES appointment but did not attend. 14 PES were delivered over 6 weeks.

Groups were observed to discuss individual experiences of their IBD, and how adalimumab had changed their lives for the better. Their main concerns were that the biosimilar might be less effective and whether they could they 'switch' back in that scenario.

Those patients happy to switch without attending PES and those who Did Not Attend PES were called by the Nurse to confirm current frequency and device used.

$125 / 132$ patients completed the switch. New prescriptions were completed, a database of biosimilar patients was set up, the old database was archived, and GP letters were dictated.

7 patients did not switch: 2 discontinued biologic (remission) 2 had surgical resection 2 switched to an alternative biologic and one refused to switch on principle and remains on Humira.

Conclusions PES can facilitate biosimilar switch in a rapid patient-centred fashion. Many patients are happy to switch without attendance. Extending the notice period may improve attendance of patients who would like to attend. PES could be extended to biologic 'new starts' too.

\section{P370 ACCEPTABILITY AND OUTCOME OF INVESTIGATING IRON DEFICIENCY ANAEMIA IN THE ELDERLY}

Alec Maynard*, Jessica Johnson, John Hebden, Stuart Riley, David Dacosta. Sheffield Teaching Hospitals NHS Foundation Trust, Sheffield, UK

\subsection{6/gutjnl-2020-bsgcampus.444}

Introduction The investigation of iron deficiency anaemia (IDA) in the over 80-year-olds presents a unique challenge due to co-morbidity, uncertain prognosis and differing attitudes to acceptability of invasive investigation. We evaluated the investigations accepted and the subsequent outcomes for elderly patients.

Methods We prospectively collected consecutive GP referrals of IDA patients aged $80+$ between 2015 and 2018 seen by a single gastroenterologist. The options of invasive investigation (bidirectional endoscopy), non-invasive investigation (CTC CT pneumocolon; CT long oral prep; CT TAP - CT thorax, abdomen, pelvis), or no investigation, were discussed and offered in a standardised manner. 